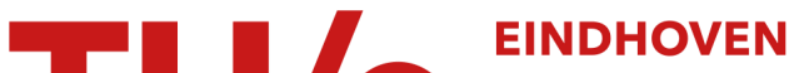 \\ UNIVERSITY OF \\ TECHNOLOGY
}

\section{Global quantitative predictions of complex laser dynamics}

Citation for published version (APA):

Wieczorek, S., Simpson, T. B., Krauskopf, B., \& Lenstra, D. (2002). Global quantitative predictions of complex laser dynamics. Physical Review E - Statistical, Nonlinear, and Soft Matter Physics, 65(4), 045207-1/4. [045207]. https://doi.org/10.1103/PhysRevE.65.045207

DOI:

10.1103/PhysRevE.65.045207

Document status and date:

Published: 01/01/2002

\section{Document Version:}

Publisher's PDF, also known as Version of Record (includes final page, issue and volume numbers)

\section{Please check the document version of this publication:}

- A submitted manuscript is the version of the article upon submission and before peer-review. There can be important differences between the submitted version and the official published version of record. People interested in the research are advised to contact the author for the final version of the publication, or visit the $\mathrm{DOI}$ to the publisher's website.

- The final author version and the galley proof are versions of the publication after peer review.

- The final published version features the final layout of the paper including the volume, issue and page numbers.

Link to publication

\section{General rights}

Copyright and moral rights for the publications made accessible in the public portal are retained by the authors and/or other copyright owners and it is a condition of accessing publications that users recognise and abide by the legal requirements associated with these rights.

- Users may download and print one copy of any publication from the public portal for the purpose of private study or research.

- You may not further distribute the material or use it for any profit-making activity or commercial gain

- You may freely distribute the URL identifying the publication in the public portal.

If the publication is distributed under the terms of Article $25 \mathrm{fa}$ of the Dutch Copyright Act, indicated by the "Taverne" license above, please follow below link for the End User Agreement:

www.tue.nl/taverne

Take down policy

If you believe that this document breaches copyright please contact us at:

openaccess@tue.nl

providing details and we will investigate your claim. 


\title{
Global quantitative predictions of complex laser dynamics
}

\author{
Sebastian Wieczorek, ${ }^{1, *}$ Thomas B. Simpson, ${ }^{2}$ Bernd Krauskopf, ${ }^{3}$ and Daan Lenstra ${ }^{1}$ \\ ${ }^{1}$ Department of Physics and Astronomy, Vrije Universiteit Amsterdam, De Boelelaan 1081, 1081 HV Amsterdam, The Netherlands \\ ${ }^{2}$ Jaycor, 3394 Carmel Mountain Road, San Diego, California 92121 \\ ${ }^{3}$ Department of Engineering Mathematics, University of Bristol, Bristol BS8 1TR, United Kingdom
}

(Received 16 May 2001; published 11 April 2002)

\begin{abstract}
We demonstrate unprecedented agreement between a theoretical two-dimensional bifurcation diagram and the corresponding experimental stability map of an optically injected semiconductor laser over a large range of relevant injection parameter values. The bifurcation diagram encompasses both local and global bifurcations mapping out regions of regular, chaotic, and multistable behavior in considerable detail. This demonstrates the power of dynamical systems modeling for the quantitative prediction of nonlinear dynamics and chaos of semiconductor lasers.
\end{abstract}

DOI: 10.1103/PhysRevE.65.045207

PACS number(s): 05.45.Gg, 05.45.Xt, 42.55.Px

It is the ultimate goal of modeling to achieve good agreement between theoretical predictions and experimental data. In this paper we compare a theoretical two-dimensional bifurcation diagram and the corresponding experimental diagram for a semiconductor laser with optical injection. Theory and experiment agree so well that quantitative prediction of complex laser dynamics are possible. This constitutes the complete verification of a model of a semiconductor laser system in terms of its complex nonlinear dynamics.

Lasers have found their way to daily-life applications, and by far the majority of lasers today are semiconductor lasers. They are small and integrable, cheap to produce, electrically pumped, and easy to modulate, have high efficiencies, but also show a number of properties generally seen as drawbacks. Due to intrinsic relaxation oscillations [1] and selfphase modulation [2], these lasers are very sensitive to external perturbations. The most notable are optical injection and external optical feedback $[3,4]$, and it is now well established that they lead to instabilities that are manifestations of highly complex structures of nonlinear dynamics and bifurcations [5].

Today dynamical instabilities in semiconductor lasers are considered for applications, such as secure communication [6,7] or dynamics based computations [8]. Moreover, the present drive towards all-optical signal manipulation in optical communication networks requires a fundamental and detailed understanding of the complexity of dynamical behaviors of semiconductor lasers and optical amplifiers.

In this respect, the single-mode semiconductor laser with optical injection is a generic system of great simplicity, a technologically important example of a forced nonlinear oscillator [4], and, at the same time, a system that holds promise for understanding more complicated systems, such as mutually coupled lasers or lasers with delayed optical feedback. It can be described by the rate equations [9]

$$
\begin{gathered}
\dot{E}=K+\left[\frac{1}{2}(1+i \alpha) n-i \omega\right] E, \\
\dot{n}=-2 \Gamma n-(1+2 B n)\left(|E|^{2}-1\right),
\end{gathered}
$$

\footnotetext{
*Email address: sebek@nat.vu.nl
}

a three-dimensional dynamical system for the complex electric field amplitude $E=E_{x}+i E_{y}$ and the population inversion $n$. The two control parameters are the injected field rate $K$ and the detuning frequency $\omega$ with respect to the freerunning laser frequency. The laser operates above its freerunning lasing threshold and Eqs. (1) are scaled such that for the free-running laser the light intensity $|E|^{2}$ and the relaxation oscillation angular frequency $\omega_{R}$ are both equal to one. The reference frame is that where the injected field has zero frequency. Parameters $B$ and $\Gamma$ are fixed and given by $B$ $=\omega_{R} / 2 \Gamma_{0}$ and $\Gamma=\Gamma_{N} / 2 \omega_{R}+B$, where $\Gamma_{0}$ is the inverse photon lifetime and $\Gamma_{N}$ the inverse electron-hole recombination time. The parameter $\alpha$ takes account of the imaginary part of the gain and is often referred to as linewidth enhancement or self-phase-modulation parameter.

System (1) produces a wealth of dynamical complexity, whose systematic investigation is still being continued. Earlier theoretical studies [10-12] focused mainly on particular phenomena occurring in localized regions of parameter space. The effects of various intrinsic laser parameters were studied with numerically calculated bifurcation diagrams [13]. Good agreement between a previous experimental stability diagram by one of the present authors [14] and singlemode rate equations was found in Ref. [19] in terms of the quantitative agreement of spectra for selected bifurcation transitions, and in Ref. [9] on the level of a unifying bifurcation picture in a large region of relevant parameter values. However, the Fabry-Perot laser used in that experiment showed mode hopping between different longitudinal modes in quite large regions, particularly near the locking boundary for negative detunings.

We present our main result in Fig. 1: the comparison of a experimental bifurcation diagram obtained for a distributed feedback (DFB) semiconductor laser operating at $1.557 \mu \mathrm{m}$, which is strictly single-mode throughout the entire range of injection values, and a theoretical diagram for this DFB laser computed with the numerical continuation package AUTO [15]. These results were not obtained separately. The experimental measurements were guided by the theoretical predictions leading to experimental identification of more phenomena such as a bistability and a chaotic intermittent transition. 


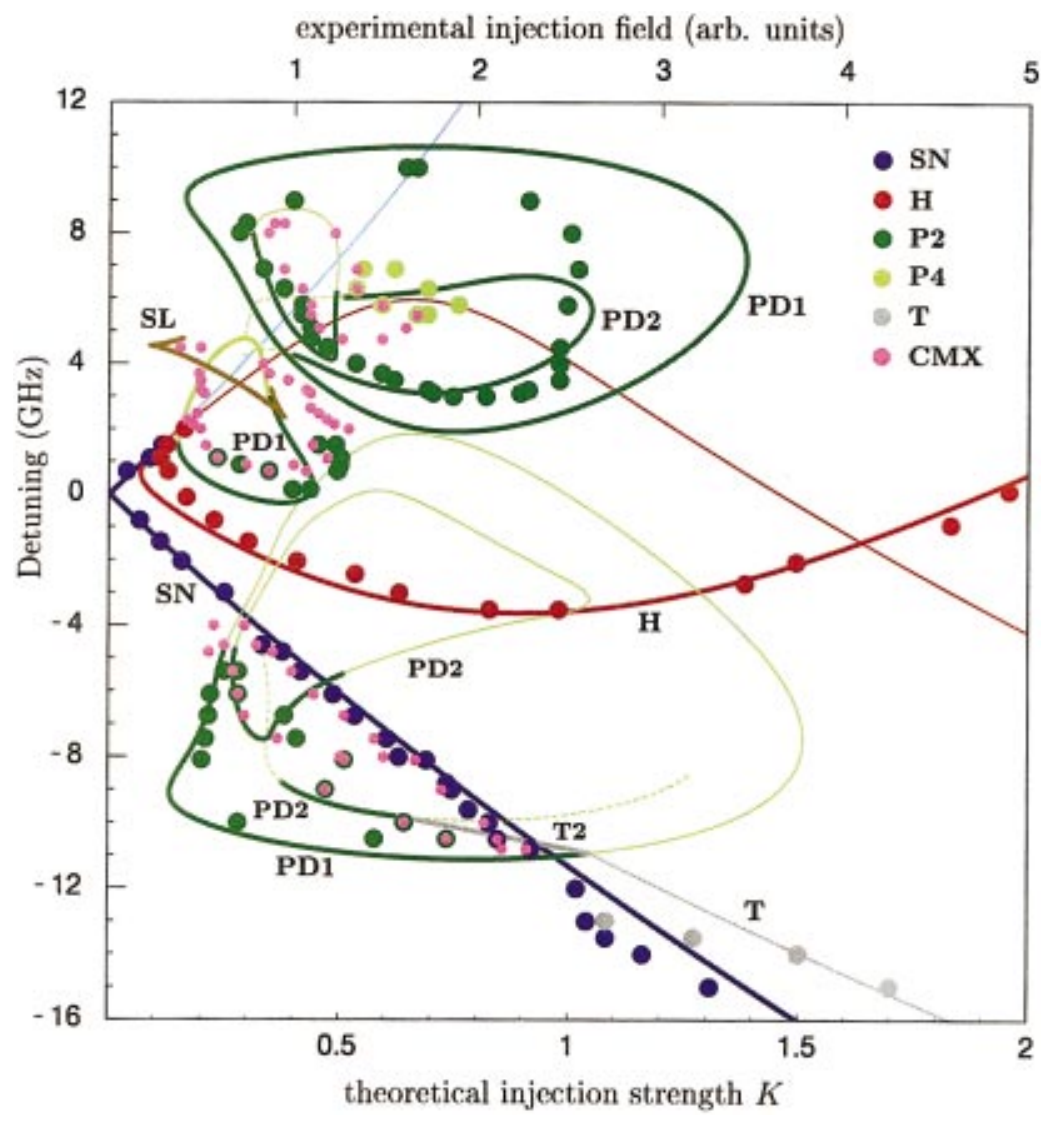

FIG. 1. (Color) Bifurcation diagram in the $(K, \omega)$ plane (plotted as curves) overlaid with the experimentally measured stability diagram (plotted as dots).
Indeed, the agreement between theory and experiment is so good that quantitative predictions of complex laser dynamics is possible.

The curves in Fig. 1 are computed from Eqs. (1) for the experimentally obtained parameter values $\alpha=2.6, \quad B$ $=0.0295$, and $\Gamma=0.0973$ specifying the DFB laser used in the experiment. Specifically, $\alpha$ was determined by measuring the asymmetry of the modulation sidebands in the optical spectra of the DFB laser subject to weak current modulation [16], while $B$ and $\Gamma$ were determined from measurements of the amplitude (output power) spectra of the laser subject to weak optical injection [17]. For the operating conditions of Fig. $1, \Gamma_{0} \approx 5 \times 10^{11} \mathrm{~s}^{-1}, \Gamma_{N} \approx 4 \times 10^{9} \mathrm{~s}^{-1}$, and $\omega_{R} \approx 29.5$ $\times 10^{9} \mathrm{rad} / \mathrm{s}$. The error estimate for $\alpha$ is approximately $-15 \%$ to $+10 \%$, while for the other parameters it is less than $\pm 10 \%$. Each curve in Fig. 1 corresponds to a specific bifurcation (a qualitative change in the behavior of the laser), and they all fit together on a global level into a consistent structure that divides the $(K, \omega)$ plane into regions of different types of laser output. Saddle-node bifurcations (SN) are in blue, Hopf bifurcations $(\mathrm{H})$ in red, period-doubling bifurcations (PD) in green, torus bifurcations (T) in gray, and saddle-node bifurcations of periodic orbits (SL) in brown. Bifurcations of stable solutions (called supercritical) are plotted as thick curves, and bifurcations of unstable solutions (called subcritical, and needed for understanding the global dynamical picture) are plotted as thinner curves. See Ref. [9] for more technical details and, e.g., Ref. [18] as a general reference to bifurcation theory.

The experimental results are shown in Fig. 1 by colored dots. For the DFB semiconductor laser we used, less than $1 \%$ (measurement uncertainty) of the total output power was contained in the side modes. Further, under optical injection the power in the side modes deviated by less than $10 \%$ from its free-running value over the range of operating conditions studied. The fiber-coupled experimental apparatus consists of an optically isolated master laser that is coupled to the slave laser through a variable attenuator and an optical circulator. Both master and slave laser are under independent current and temperature control. The slave laser was biased at twice its lasing threshold, and its output is mixed with the frequency-tunable output from a third laser that acts as a local oscillator and then detected by a photodiode. As the local oscillator's optical frequency is tuned across the range of optical frequencies generated by the slave laser, the optical spectrum is measured using a microwave spectrum analyzer. Frequency resolution is better than $100 \mathrm{MHz}$ and the dynamic range is greater than $50 \mathrm{~dB}$. Optical spectra, like those shown below, are used to identify operating conditions where stable, periodic, and more complex operating characteristics are observed [14,19]. The offset frequency of the master laser with respect to the slave laser is adjusted by changing its operating temperature. The injected power is adjusted by changing the variable attenuator. An experimental bifurcation diagram is generated by stepping the master laser through operating points of interest and recording the optical spectra generated by the slave laser under optical injection.

In Fig. 1 each dot indicates an observed qualitative change of spectral characteristics. The blue dots correspond 
to the transition from stable, locked operation to unlocked operation while gray dots at large negative detunings correspond to transitions from unlocked to stable locked operation. Red dots denote the appearance of the relaxation oscillation. Dark green dots bound regions of period-two operation, light green dots confine regions of period-four oscillation and small pink dots bound regions of more complicated spectral and dynamic characteristics. Small pockets of periodic dynamics in these regions are not shown. To absolutely compare model and experiment, it would be necessary to measure the power from the master laser that is actually coupled into the oscillating mode of the slave laser, not simply measure the power incident on the laser facet. Because this was not possible, we used the experimentally observed locking-unlocking transition and the calculated saddle-node curve as the calibration reference.

Clearly, several curves in Fig. 1 show very good agreement with the respective measurements within a few percent, while particularly in the upper region bounded by perioddoublings the agreement is less spectacular, but still on the order of about $25 \%$. While the bifurcation diagram comprises many different physical phenomena that depend on different parameters that are often difficult to estimate accurately, the agreement is well within the accuracy of the experimental determination of the laser parameters. This is why we claim that Fig. 1 demonstrates overall quantitative agreement between theory and experiment. Furthermore, the agreement is so good that it allows for quantitative predictions of complex dynamics.

Let us now discuss the general structure of Fig. 1 in more detail. In the locking region, confined between the two curves SN and $H$ in Fig. 1, the laser emits light exactly at the frequency of the injected light. As the locking region is approached from below for $K<1$, the slave laser emits an oscillating signal (due to the beating between the master and slave fields) until SN is reached where a stable equilibrium appears in a saddle-node bifurcation (for $K>1$ there is a bistability discussed hereafter). Between $\mathrm{SN}$ and $\mathrm{H}$ the slave laser locks to the injected field. Along curve $\mathrm{H}$, the stable equilibrium loses its stability in a Hopf bifurcation, and a sustained relaxation oscillation is found above $\mathrm{H}$. A periodic orbit, of "basic" period $\approx 2 \pi / \omega_{R}$ at low injection levels and monotonically decreasing period at higher injection levels, then appears and the laser produces a stable intensity oscillation.

Periodic solutions may encounter further instabilities. Figure 1 shows three regions bounded by period-doubling bifurcations PD1 of the basic periodic orbit, one for negative detuning $\omega$, and a small and a bigger region for positive $\omega$. Transitions from period-two to period-four operation occur along the curves PD2.

The period-doubling curves for negative $\omega$ agree well with the measurements, to within a few percent. Furthermore, the pink dots, signaling a change to chaotic dynamics in the measured spectra, agree very well with the theoretical boundary of complicated dynamics given by the accumulation of period-doubling curves and the locking range [20]. Where the lower period-doubling curve PD1 changes from supercritical to subcritical, a curve of torus bifurcations
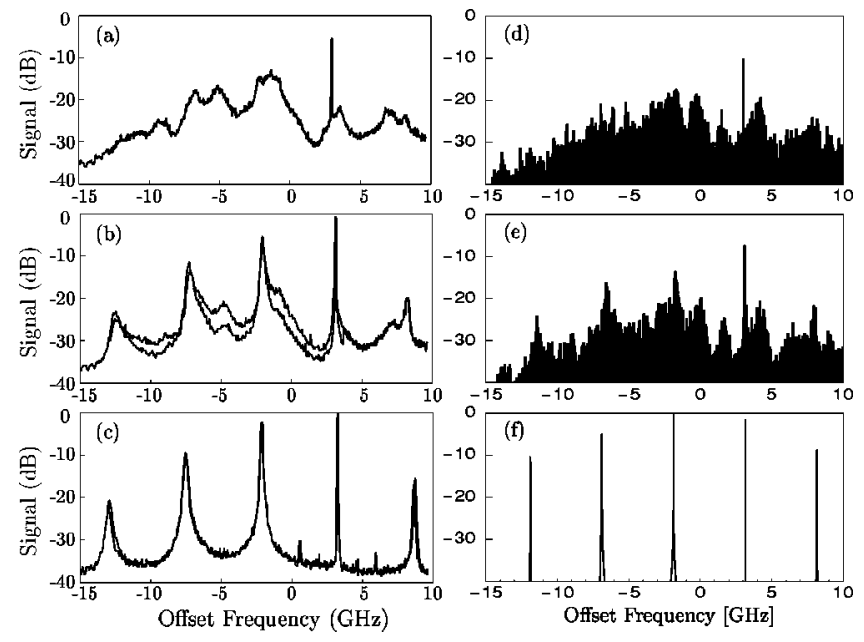

FIG. 2. Experimental (left column; each panel shows two traces of the spectrum) and theoretical (right column) spectra showing an intermittent transition. The experimental injection field $\approx 1$ and from (a) to (c) $\omega$ takes the values $3,3.13$, and $3.2 \mathrm{GHz}$. For the theoretical spectra $K=0.31$ and from (d) to (f) $\omega$ takes the values 3, 3.11 , and $3.15 \mathrm{GHz}$. The Offset Frequency is given with respect to the solitary laser frequency.

emerges, leading to quasiperiodic or synchronized oscillations, depending on the ratio of the two frequencies involved. An attracting torus is born along the thick gray curve $\mathrm{T} 2$ and then breaks up into a chaotic attractor [21]. This leads to the chaotic dynamics above $\mathrm{T} 2$ detected experimentally in this region (pink dots). The thin gray torus bifurcation curve $\mathrm{T}$, on the other hand, bounds a region of bistability between the running phase solution and the locked solution; this was found previously in Ref. [22], numerically investigated in Ref. [24], and theoretically studied in Refs. [25,20]. This region opens at $\approx-12 \mathrm{GHz}$ and its other boundary is $\mathrm{SN}$. The upper bistability boundary was found experimentally (gray dots) to within a few percent of the theoretical curve T. Crossing across the blue curve $\mathrm{SN}$ and the thin gray curve $\mathrm{T}$ indeed leads to a hysteresis loop in the experiment as predicted. This region of bistability was not found in the FabryPerot laser used in Ref. [14] due to mode hopping in this region of the $(K, \omega)$ plane.

For the small central region of period-doubling and complex dynamics the agreement between theory and experiment is good, to within $10 \%$. Again, the green and pink dots agree well with the region bounded by the theoretical bifurcation curves. The chaotic region inside the curves PD1 and PD2 can be entered (or exited) in different manners, and one is an intermittent transition from periodic oscillations directly to chaos caused by a global saddle-node of periodic orbit bifurcation taking place on a chaotic attractor [23]. This happens along the brown curve SL. Experimentally, this chaotic region is confined from above by pink dots, already suggesting an abrupt transition out of chaos. Detailed measurements of spectra in this region, shown in the left column of Fig. 2, reveal an intermittent transition. The chaotic signal in Fig. 2(a) changes abruptly to oscillations in Fig. 2(c). Very close to the bifurcation value, one sees fluctuations of the spectrum between the two cases due to parameter jitter, as is shown by 
the two overlaid spectra in Fig. 2(b). The experimental spectra reveal structures that agree very well with those computed from Eqs. (1), shown in the right column of Fig. 2. Notice in Figs. 2(d) and 2(e) that the chaotic attractor anticipates a periodic orbit that is about to appear, as demonstrated by the development of the narrow equidistant peaks in the spectra. Then, at the saddle-node of periodic orbit bifurcation SL, two new periodic orbits appear, one attracting and one a saddle orbit. The laser makes a sudden transition from chaotic to periodic oscillations in Fig. 2(f). An abrupt transition was observed in previous experiments $[14,19]$ but then it was unclear whether the effect was genuine or due to multimode laser operation. This shows the value of bifurcation diagrams as a tool to find and interpret complicated transitions in this laser system.

The deviation between theory and experiment is not exceeding about $25 \%$ for the larger region of period-doubling and complex dynamics. In particular, the pink dots indicating transition to complex dynamics are in good agreement with the theoretical prediction. It is unclear why the deviation between model and experiment should be greatest for positive detunings at large injection levels. It is known that $\alpha$ is a critical parameter in determining the nonlinear dynamics. Further, optical injection induces changes in the carrier density and there is some evidence that $\alpha$ may be sensitive to the changes [16]. Indeed, the model rests on the fact that the complex gain can be linearly related to the carrier density, spatially averaged over the laser structure. It is extraordinary how well this simplification holds for this laser, given the variations in the optical field and carrier density over the structure.

In conclusion, for the nonlinear dynamical behavior of a semiconductor laser with optical injection we have presented a comparison between an experiment using a single-mode DFB semiconductor laser and bifurcation theory based on Eqs. (1). Very good agreement, not only locally but also globally, has been obtained in unprecedented detail. Furthermore, we have used the model to make quantitative predictions about details of the nonlinear dynamics, specifically determining the detuning and injection levels required for the bistability, and the mechanism and characteristics of an intermittent transition to chaos. This demonstrates predictive and quantitative modeling of nonlinear laser dynamics, and it constitutes the state of the art in dynamical systems modeling of a complex system.

The research of S.W. was supported by the Foundation for Fundamental Research on Matter (FOM), which is financially supported by the Netherlands Organization for Scientific Research (NWO). The work of T.B.S. was supported, in part, by the U.S. Army Research Office under Contract No. DAAG-55-98-C-0038.
[1] H. Haken and H. Sauerman, Z. Phys. 176, 47 (1963).

[2] K. Petermann, Laser Diode Modulation and Noise (Kluwer Academic, Dordrecht, The Netherlands, 1988).

[3] J. Sacher, D. Baums, P. Panknin, W. Elsäßer, and E.O. Göbel, Phys. Rev. A 45, 1893 (1992).

[4] G.H.M. van Tartwijk and G.P. Agrawal, Prog. Quantum Electron. 22, 43 (1998).

[5] B. Krauskopf and D. Lenstra, Fundamental Issues of Nonlinear Laser Dynamics: Concepts, Mathematics, Physics and Applications, AIP Conf. Proc. No. 548 (AIP, Melville, 2000).

[6] C.R. Mirasso, P. Colet, and P. Garcia-Fernández, IEEE Photonics Technol. Lett. 8, 299 (1996).

[7] G.D. Van Wiggeren and R. Roy, Science 279, 1198 (1997).

[8] S. Sinha and W.L. Ditto, Phys. Rev. Lett. 81, 2156 (1998).

[9] S. Wieczorek, B. Krauskopf, and D. Lenstra, Opt. Commun. 4172, 279 (1999).

[10] J.R. Tredicce, F.T. Arecchi, G.L. Lippi, and G.P. Puccioni, J. Opt. Soc. Am. B 2, 173 (1985).

[11] H.G. Solari and G.L. Oppo, Opt. Commun. 111, 173 (1994).

[12] T. Erneux, V. Kovanis, A. Gavrielides, and P.M. Alsing, Phys. Rev. A 53, 4372 (1996).

[13] S.K. Hwang and J.M. Liu, Opt. Commun. 183, 195 (2000).

[14] T.B. Simpson, J.M. Liu, K.F. Huang, and K. Tai, Quantum Semiclassic. Opt. 9, 765 (1997).
[15] E. Doedel, T. Fairgrieve, B. Sandstede, A. Champneys, Yu. Kuznetsov, and X. Wang, AUTO 97 Continuation and bifurcation software for ordinary differential equations, http://indy.cs. concordia.ca/auto/main.html

[16] T.B. Simpson, F. Doft, E. Strzelecka, J.J. Liu, W. Chang, and G.J. Simonis, IEEE Photonics Technol. Lett. 13, 776 (2001).

[17] J.M. Liu and T.B. Simpson, IEEE J. Quantum Electron. 30, 957 (1994).

[18] Yu. A. Kuznetsov, Elements of Applied Bifurcation Theory, Applied Mathematical Sciences Vol. 112 (Springer, New York, 1995).

[19] V. Kovanis, A. Gavrielides, T.B. Simpson, and J.M. Liu, Appl. Phys. Lett. 67, 2780 (1995).

[20] S. Wieczorek, B. Krauskopf and D. Lenstra, Opt. Commun. 183, 215 (2000).

[21] B. Krauskopf, S. Wieczorek, and D. Lenstra, Appl. Phys. Lett. 77, 1611 (2000).

[22] R. Hui, A. D’Ottavi, A. Mecozzi, and P. Spano, IEEE J. Quantum Electron. 27, 1688 (1991).

[23] S. Wieczorek, B. Krauskopf, and D. Lenstra, Phys. Rev. E 64, 056204 (2001).

[24] J. M. Liu and T. B. Simpson, IEEE J. Quantum Electron. 30, 957 (1994).

[25] V. Kovanis, T. Erneux, and A. Gavrielides, Opt. Commun. 159, 177 (1999). 